\title{
C-terminal tensin-like protein mediates invasion of human lung cancer cells and is regulated by signal transducer and activator of transcription 3
}

\author{
Daine T. Bennett, MD, Thomas Brett Reece, MD, Lisa S. Foley, MD, Ayla Sjoberg, HSD, \\ Xianzhong Meng, MD, PhD, David A. Fullerton, MD, and Michael J. Weyant, MD
}

\begin{abstract}
Objectives: C-terminal tensin-like (Cten) protein, a component of focal adhesions, contributes to cell motility and invasion in multiple human cancers. Epidermal growth factor can activate signal transducer and activator of transcription 3, and both contribute to invasion through focal adhesion interactions. We hypothesize that Cten may mediate invasion of lung cancer cells provided by epidermal growth factor via signal transducer and activator of transcription 3 .
\end{abstract}

\begin{abstract}
Methods: Four human non-small cell lung cancer cell lines were treated with epidermal growth factor to evaluate activation of the signal transducer and activator of transcription 3 pathway and induction of Cten expression. Chemical inhibition of signal transducer and activator of transcription 3 was used to evaluate the effect on epidermal growth factor-induced Cten expression. Protein expression was quantified by Western blot. H125 and A549 cells were transduced with short-hairpin RNA via lentiviral vector to knockdown expression of Cten. An in vitro transwell invasion assay was used to assess the effects of Cten knockdown on cell invasion ( $\mathrm{n}=3$ for all experiments).
\end{abstract}

Results: Stimulation of lung cancer cells with epidermal growth factor activated the signal transducer and activator of transcription 3 pathway and induced expression of Cten in all cell lines. Signal transducer and activator of transcription 3 inhibition significantly reduced epidermal growth factor-induced expression of Cten in H125 $(P<.0001)$, H358 $(P=.006)$, and H441 $(P=.014)$ cells in a dose-dependent manner. Knockdown of Cten expression resulted in significant decreases in cellular invasion in both H125 $(P=.0036)$ and A549 $(P=.0006)$ cells.

Conclusions: These are the first findings in lung cancer to demonstrate that Cten expression mediates invasion of human lung cancer cells and is upregulated by epidermal growth factor via signal transducer and activator of transcription 3 pathway. Cten should be considered a potential therapeutic target for lung cancer. (J Thorac Cardiovasc Surg 2015;149:369-75)

See related commentary on pages 376-7.

Lung cancer remains the leading cause of cancer-related death in the United States, claiming more lives than the next 3 leading types of cancer combined. With a dismal overall 5 -year survival of only $16 \%$, patients with lung

\footnotetext{
From the Division of Cardiothoracic Surgery, Department of Surgery, University of Colorado Denver, School of Medicine, Aurora, Colo.

Disclosures: Michael J. Weyant reports consulting fees from Myriad. The other authors have nothing to disclose with regard to commercial support.

Read at the 40th Annual Meeting of The Western Thoracic Surgical Association, Dana Point, California, June 25-28, 2014.

Received for publication June 25, 2014; revisions received Aug 4, 2014; accepted for publication Aug 24, 2014; available ahead of print Oct 16, 2014.

Address for reprints: Michael J. Weyant, MD, Division of Cardiothoracic Surgery, Department of Surgery, University of Colorado Denver School of Medicine, 12631 East 17th Ave, MS C310, Aurora, CO 80045 (E-mail: michael.weyant@ ucdenver.edu).

0022-5223/\$36.00

Copyright (c) 2015 Published by Elsevier Inc. on behalf of The American Association for Thoracic Surgery

http://dx.doi.org/10.1016/j.jtcvs.2014.08.087
}

cancer urgently need improved treatment strategies to combat this deadly malignancy. These poor survival outcomes in non-small cell lung cancer result, in part, from a high rate of metastatic disease at the time of diagnosis.

Continued investigation into the molecular mechanisms of lung cancer invasion and metastasis could identify potential therapeutic targets to prevent or stabilize metastatic disease burden. One well-established mechanism through which cancer cells metastasize is via dysregulation of focal adhesion complexes. ${ }^{2}$ Focal adhesion complexes involve transmembrane integrin proteins that interact with more than 50 different structural and signaling proteins. These adhesion complexes interact with both the cellular cytoskeleton and the extracellular matrix. The mechanisms of dysregulation of focal adhesions include remodeling of the actin-cytoskeleton to form lamellipodia with associated reshuffling of focal adhesions toward the leading edge of a migrating cell. ${ }^{2}$ When these focal adhesions become dysregulated, cancer cells acquire a motile, invasive phenotype ultimately leading to metastasis. 


$$
\begin{aligned}
& \text { Abbreviations and Acronyms } \\
& \begin{aligned}
\text { Cten }= & \text { C-terminal tensin-like } \\
\text { EGF }= & \text { epidermal growth factor } \\
\text { shRNA }= & \text { short-hairpin RNA } \\
\text { STAT3 }= & \text { signal transducer and activator of } \\
& \text { transcription } 3
\end{aligned}
\end{aligned}
$$

C-terminal tensin-like (Cten) protein, also referred to as "tensin-4," incorporates into the cytoplasmic side of focal adhesion complex by C-terminal binding to integrin proteins. Unlike other members of the tensin family, the truncated Cten lacks an N-terminal, actin-binding domain. ${ }^{3}$ Breast and melanoma tumors that stain strongly positive for Cten demonstrate worse 5-year survival. ${ }^{4,5}$ Cten mRNA expression has been shown to correlate with advanced tumor stage in lung cancer. ${ }^{6}$ Elevated Cten expression recently has been shown to correlate with increased metastatic properties ${ }^{7-10}$ in a number of in vitro and in vivo solid organ tumor models; however, the role of Cten in the invasive properties of lung cancer has not been evaluated.

Signal transducer and activator of transcription 3 (STAT3) is a driver of lung cancer progression. ${ }^{11}$ Stimulation of cancer cells with epidermal growth factor (EGF) can activate the STAT3 pathway directly or through the EGF receptor. ${ }^{12}$ EGF also was shown to induce Cten expression in colorectal cancer cells. ${ }^{13}$ During activation, STAT3 forms a homodimer after phosphorylation of tyrosine residues and translocates to the nucleus to regulate transcription. The transcriptional capacity is fully optimized after phosphorylation of the serine residue. ${ }^{14}$ In breast cancer cells, Cten expression was shown to be dependent on the STAT3 pathway. $^{7}$

The importance of Cten in the malignant properties of non-small cell lung cancer has yet to be investigated, although the relation among EGF, lung cancer growth, and invasion makes this an attractive target of study. Given the data from other solid organ tumors demonstrating that Cten plays a role in the invasion of cancer cells, we hypothesized that knockdown of Cten expression would reduce the invasive capacity of non-small cell lung cancer cells. We demonstrate for the first time that knockdown of Cten expression reduces invasion in human lung cancer cells and that the STAT3 pathway is a regulator of Cten expression.

\section{MATERIALS AND METHODS \\ Cell Culture and Materials}

Human non-small cell lung cancer cell lines H125, H358, H441, and A549 were used for all experiments. H125 cells were purchased from the University of Colorado Cancer Center Tissue Culture Core (Aurora, Colo). H358 and H441 cells were a gift from Dr Kim O'Neill (Provo,
Utah). A549 cells were purchased from American Type Culture Collection (Manassas, Va). H125, H358, and H441 cells were maintained in Roswell Park Memorial Institute (Life Sciences, Grand Island, NY), and A549 cells were cultured in Ham's F12 (Corning, Manassas, Va). Medium was supplemented with $10 \%$ fetal bovine serum and $1 \%$ penicillin-streptomycin for standard culture conditions. Cells were grown in $75 \mathrm{~cm}^{2}$ tissue culture flasks (Corning) in a humidified incubator at $37^{\circ} \mathrm{C}$ and $5 \%$ carbon dioxide.

Cten antibody was purchased from Sigma-Aldrich (St Louis, Mo). Antibodies for phospho-STAT3, total-STAT3, and glyceraldehyde 3-phosphate dehydrogenase antibody were purchased from Cell Signaling (Beverly, Mass). EGF was purchased from BD Biosciences (Franklin Lakes, NJ) and reconstituted in sterile water. STAT3 inhibitor (S3i-201) was purchased from Sigma-Aldrich and reconstituted in dimethyl sulfoxide (Sigma-Aldrich).

\section{Cell Treatments}

Cells were plated at a density of $1 \times 10^{5}$ cells per well in 6-well plates and cultured for 24 hours. They were then serum-starved for 24 hours using medium supplemented with $0.5 \%$ fetal bovine serum. Cells were treated as described with S3i-201, EGF, or both in serum-starved medium. The STAT3 inhibitor, S3i-201, was applied at the described doses, and the dimethyl sulfoxide concentration $(0.1 \%)$ was normalized across all control and treatment wells. The inhibitor was applied for 2 hours before EGF application.

\section{Western Blotting}

At the completion of treatment times, cells were washed twice with phosphate-buffered saline and lysed in Laemelli buffer (Bio-Rad, Hercules, Calif). Proteins were separated by sodium dodecyl sulfate polyacrylamide gel electrophoresis and transferred to nitrocellulose membranes. Membranes were blocked in 5\% nonfat milk dissolved in $1 \times$ Tris-buffered saline, $0.1 \%$ Tween-20 (Sigma-Aldrich). Primary antibodies were dissolved in $4 \%$ bovine serum albumin (Sigma-Aldrich) in $1 \times$ Tris-buffered saline, $0.1 \%$ Tween- 20 . Secondary antibodies were prepared in $5 \%$ nonfat milk in $1 \times$ Tris-buffered saline, $0.1 \%$ Tween20. Membranes were developed using Pierce ECL Chemiluminescent (Thermo Fisher Scientific, Rockford, Ill). Protein quantification was performed by densitometric analysis using ImageJ Software (National Institutes of Health, Bethesda, Md). Histograms depict mean expression of protein of interest relative to control protein with standard error of the mean

\section{Short-Hairpin RNA Knockdown of C-Terminal Tensin-Like Expression}

H125 and A549 cells were plated in 6-well culture plates with standard medium for 24 hours. The medium was removed and replaced with medium containing pLKO.1 puromycin-resistant lentiviral vectors containing a short-hairpin (sh)RNA sequence targeting Cten or a nontargeted shRNA used as a control. Polybrene (Sigma-Aldrich) was also added at $8 \mu \mathrm{g} / \mathrm{mL}$ to facilitate transfection. After 24 hours of exposure to the lentiviral constructs, the medium was replaced with standard medium with puromycin (Sigma-Aldrich) at a concentration of $2 \mu \mathrm{g} / \mathrm{mL}$ to kill any nontransfected cells. Lentiviral constructs were acquired from Functional Genomics, University of Colorado at Boulder (Boulder, Colo). The shRNA sequence used for Cten knockdown was CCGGCCTTGACTCCTACATTGACTTCTC GAGAAGTCAATGTAGGAGTCAAGGTTTTTTG, which is a validated sequence from Sigma-Aldrich.

\section{In Vitro Invasion Assay}

Matrigel invasion assay (BD Biosciences, San Jose, Calif) was used to assess the effects of Cten shRNA knockdown on the ability of lung cancer cells to invade through a basement membrane in an in vitro model. This 
H125
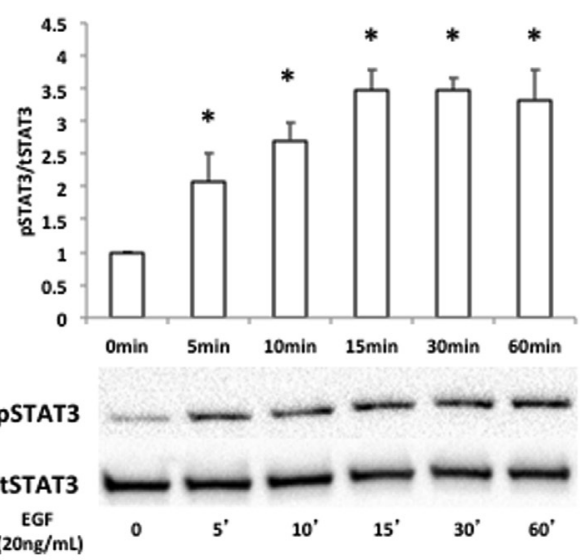

A

H441

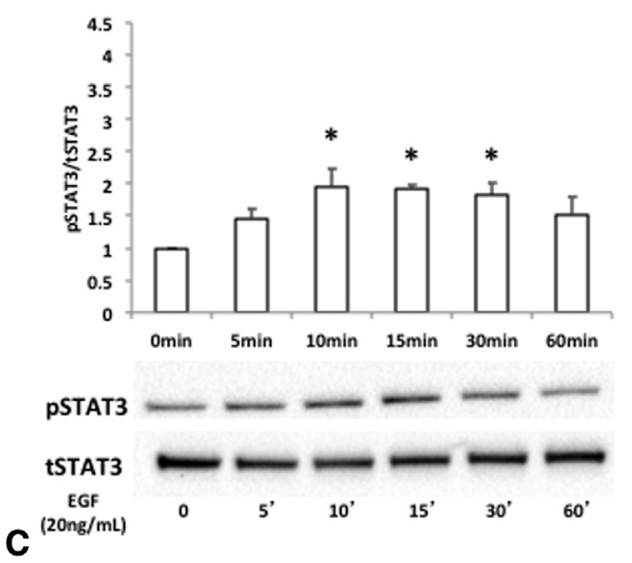

H358

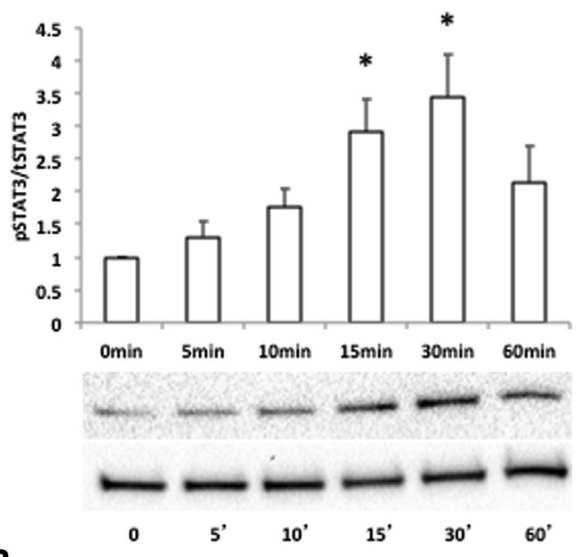

A549

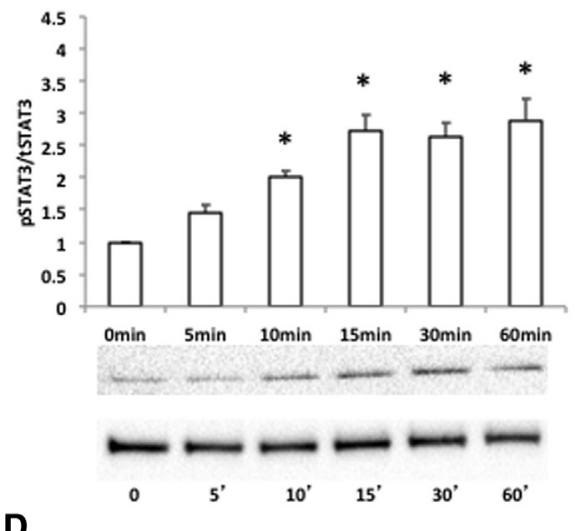

FIGURE 1. EGF activates STAT3 pathway. Cells from all 4 cell lines were treated with EGF at a concentration of $20 \mathrm{ng} / \mathrm{mL}$ over a time course from 0 to 60 minutes. Activation of the STAT3 pathway was evaluated by Western blot. Phosphorylation of STAT3 at serine 727 was quantified relative to total STAT3. STAT3 activation was significant in all 4 cell lines within 15 minutes: $\mathrm{H} 125(P=.007)(\mathrm{A}), \mathrm{H} 358(P=.0123)(\mathrm{B}), \mathrm{H} 441(P=.02)(\mathrm{C})$, and A549 $(P=.0003)$ (D). $\mathrm{n}=3$ for each cell line. EGF, Epidermal growth factor; $p S T A T 3$, phosphorylation of signal transducer and activator of transcription 3; tSTAT3, total signal transducer and activator of transcription 3.

transwell, 24-well assay consists of 2 chambers per well. The upper chamber contains a porous membrane $(8 \mu \mathrm{m})$ covered with a thin layer of Matrigel basement membrane substrate. Cells are added to the upper chamber, and it is inserted into the lower chamber, which contains a chemoattractant. Cells are allowed to incubate for a period of time, and then the porous membrane is removed and stained. Invading cells are then counted by light microscopy.

In preparation for plating the cells, the upper chambers containing the basement membrane were allowed to thaw at room temperature for 2 hours and then rehydrated with serum-free medium at $37^{\circ} \mathrm{C}$ for 2 hours. For both H125 and A549 cell lines, the untransfected, control-shRNA, and Cten-shRNA cells were each plated at a density of $5 \times 10^{4}$ in $500 \mu \mathrm{L}$ of serum-starved medium into the upper chamber. This upper chamber was inserted into the lower chamber, which contained $750 \mu \mathrm{L}$ of serumstarved medium supplemented with EGF $(50 \mathrm{ng} / \mathrm{mL})$ as a chemoattractant. The cells are allowed to invade at $37^{\circ} \mathrm{C}$ with $5 \%$ carbon. Previous work indicated that optimal incubation times for H125 and A549 cell lines were 40 and 18 hours, respectively. At the end of incubation, membranes were washed, stained, and mounted on slides according to the manufacturer's guidelines. Invading cells were counted under $20 \times$ magnification with light microscopy by a blinded observer. Seven fields were observed for each membrane, and the average number of invading cells per field was reported.

\section{Statistical Analysis}

Analysis of variance was used for comparison of means of more than 2 groups. No multiple comparisons were performed. StatView V5.0 (1998; SAS institute Inc, Cary, NC) was used for all statistical analysis.

\section{RESULTS}

Epidermal Growth Factor Activates Signal

Transducer and Activator of Transcription 3 Pathway in Non-Small Cell Lung Cancer

The ability of EGF to activate the STAT3 pathway was assessed by Western blot. For each cell line, cells were plated in $1 \mathrm{~mL}$ of standard medium in a 12 -well plate. After 24 hours of growth, standard medium was replaced with serum-starved medium for an additional 24 hours. Cells were then treated with EGF $(20 \mathrm{ng} / \mathrm{mL})$ over a time-course from 0 to 60 
H125
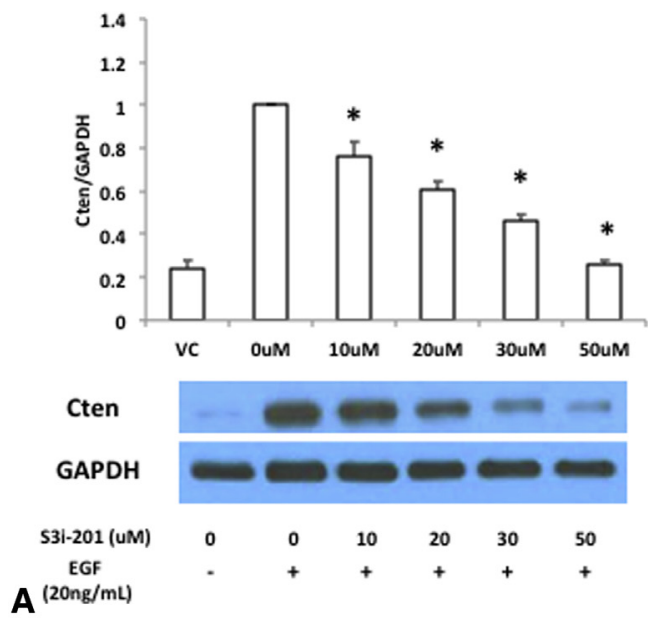

H441
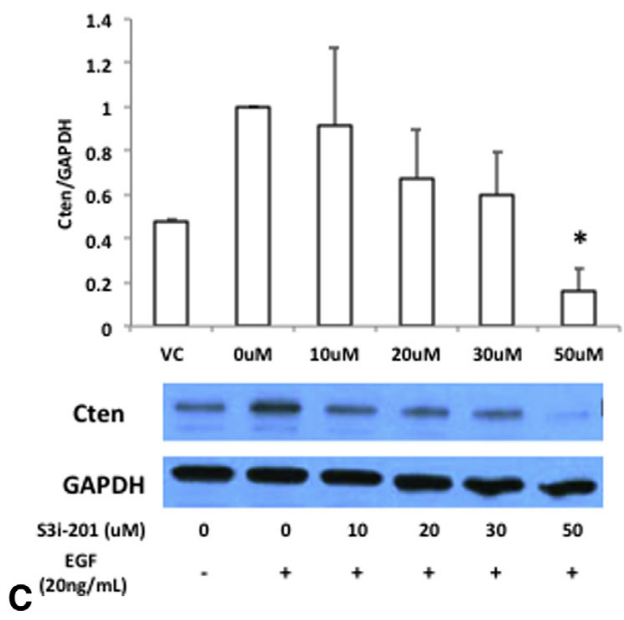

H358
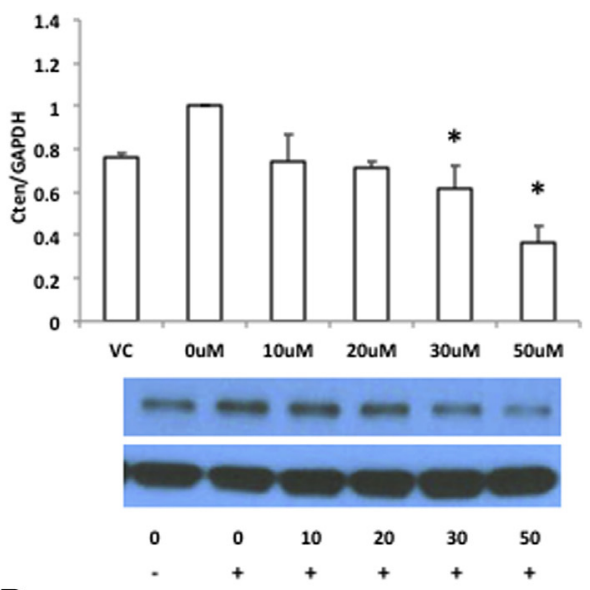

B

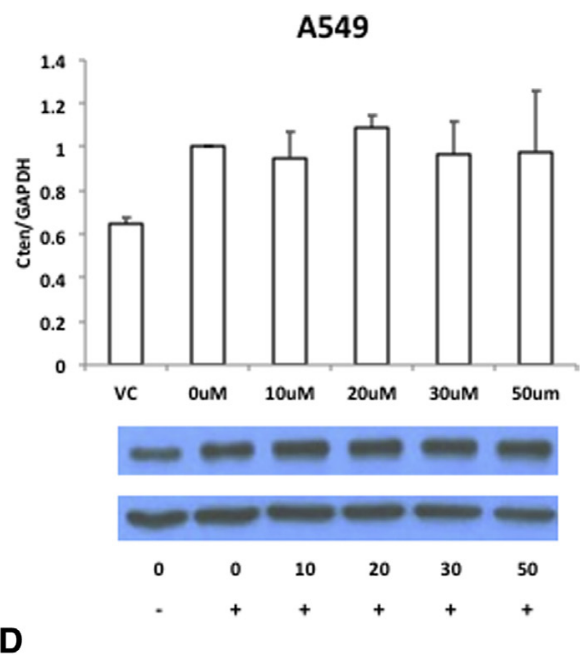

FIGURE 2. Inhibition of STAT3 abrogates EGF-induced CTEN expression. Cells were pretreated with increasing concentrations of STAT3 inhibitor (S3i-201) or the equivalent concentration of dimethyl sulfoxide $(0.1 \%)$ for 1 hour. EGF was then added to the indicated wells. Cells were cultured for an additional 48 hours and then lysed. Protein expression of Cten was normalized to glyceraldehyde 3-phosphate dehydrogenase antibody as evaluated by Western blot. EGF induced Cten expression in all 4 cell lines. STAT3 inhibition reduced Cten expression in a dose-dependent fashion in $\mathrm{H} 125(P<.0001)(\mathrm{A}), \mathrm{H} 358(P=.002)(\mathrm{B})$, and $\mathrm{H} 441$ $(P=.007)(\mathrm{C})$. Although EGF induced expression of Cten in A549 cells (D), STAT3 inhibition was ineffective in reducing Cten expression. $\mathrm{n}=3$ for each cell line. Cten, C-terminal tensin-like; $E G F$, epidermal growth factor; $G A P D H$, glyceraldehyde 3-phosphate dehydrogenase; $V C$, vehicle control.

minutes. The STAT3 pathway was activated in all 4 cell lines. Induction of phospho-STAT3 was significant within 15 minutes of EGF stimulation for H125 $(P=.007)$, H358 $(P=.0123)$, H441 $(P=.02)$, and A549 $(P=.0003)$ cells as demonstrated in Figure 1 by Western blot.

The data shown demonstrate phosphorylation of the serine $^{727}$ residue. As discussed earlier, activation of the STAT3 pathway requires phosphorylation of tyrosine ${ }^{705}$ residues and dimerization followed by activation of the serine $^{727}$ residue for maximal transcriptional activity. ${ }^{12,14}$ As has been shown in lung cancer studies, ${ }^{15}$ we found that some of the cell lines evaluated had high baseline levels of tyrosine phosphorylation with minimal induction on EGF stimulation (data not shown). However, EGF activated the serine ${ }^{727}$ residue in all cell lines, which is associated with maximal transcriptional activity of STAT3.

\section{Chemical Inhibition of Signal Transducer and Activator of Transcription 3 Abrogates Epidermal Growth Factor-Induced Cten Protein Expression}

Cells were plated in $2 \mathrm{~mL}$ of medium in 6-well plates, incubated for 24 hours in standard medium, and serumstarved for 24 hours. The specific STAT3 inhibitor, S3i-201, was applied to the cells in increasing concentrations from 10 to $50 \mu \mathrm{mol}$. Two wells were left untreated controlling for dimethyl sulfoxide concentration of $0.1 \%$. After 2 hours of pretreatment with STAT3 inhibitor, EGF $(20 \mathrm{ng} / \mathrm{mL})$ was added to all treatment wells and one of the control wells. After 48 hours of EGF treatment, all cells 


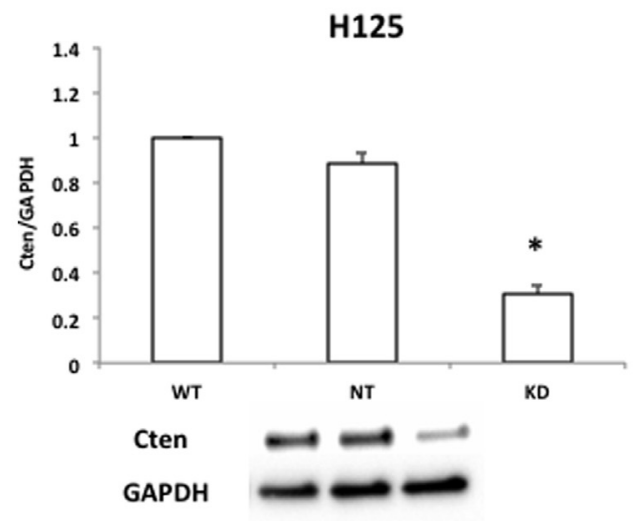

A

WT NT KD

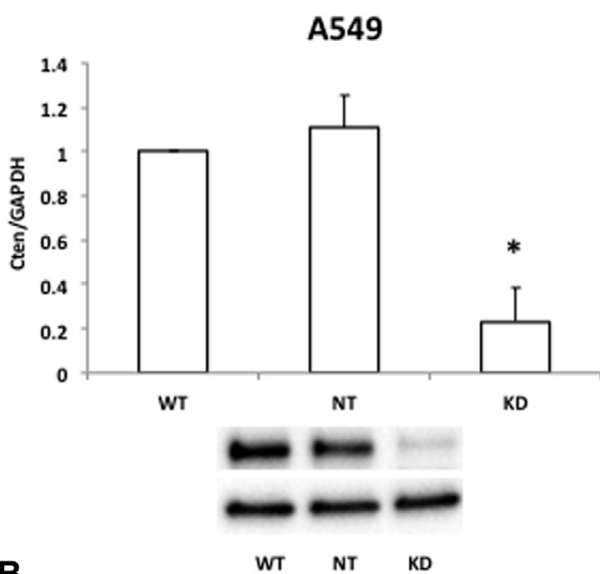

B

WT NT KD

FIGURE 3. shRNA transduction successfully reduces Cten protein expression in human lung cancer cell lines. shRNA targeting Cten expression (knockdown) or nontargeting control vector was transfected into H125 (A) and A549 (B) cell via a pLKO.1, puromycin-resistant lentiviral vector. Cten expression was successfully knocked down by at least $70 \%$ in the knockdown cells relative to the untransfected cells with minimal effects in the nontargeting control cell line for both H125 $(P<.0001)$ and A549 $(P=.0048)$ cell lines. This knockdown of Cten was stable over multiple passages of the cells. $\mathrm{n}=3$ for each cell line. Cten, Cterminal tensin-like; GAPDH, glyceraldehyde 3-phosphate dehydrogenase; KD, knockdown vector; $N T$, nontargeting control; WT, wild type control.

were lysed and protein expression of Cten was evaluated by Western blot. Figure 2 demonstrates an induction of Cten with EGF treatment alone for all 4 cell lines. STAT3 inhibition resulted in a significant, dose-dependent decrease in Cten expression in H125 $(P<.0001)$, H358 $(P=.002)$, and $\mathrm{H} 441(P=.007)$ cells (Figure 2, $A-C)$. Of note, STAT3 inhibition did not appear to have an effect on the level of EGF-induced Cten expression in the A549 cell line $(P=.407)$ (Figure $2, D)$.

\section{Successful Knockdown of C-Terminal Tensin-Like Expression With Short-Hairpin RNA}

H125 and A549 cells were transfected with a pLKO.1 lentiviral vector containing a puromycin-resistant gene and a validated shRNA sequence targeting Cten expression. The transduction of shRNA successfully reduced the protein expression of Cten by at least $70 \%$ in both H125 $(P<.0001)$ and A549 $(P=.0048)$ cells with minimal effects on cells transfected with control-shRNA. This knockdown of Cten expression was stable over multiple passages, as demonstrated by Western blot in Figure 3.

\section{C-Terminal Tensin-Like Knockdown Results in Decreased Capacity for Invasion in Lung Cancer Cells}

After establishing stable Cten-knockdowns of H125 and A549 cell lines, an in vitro transwell Matrigel invasion assay was performed to evaluate the effects of reduced Cten expression on the ability of lung cancer cells to invade. Cells transduced with Cten-shRNA demonstrated a significant reduction in their ability to invade relative to the control-shRNA cells and untransfected cells. This

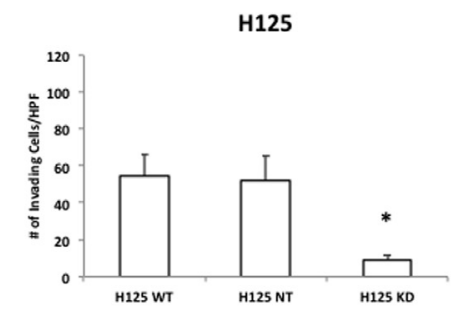

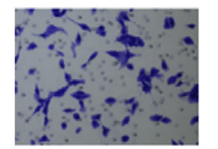

A

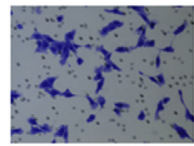

NT

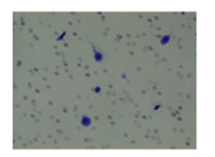

KD

\section{B}

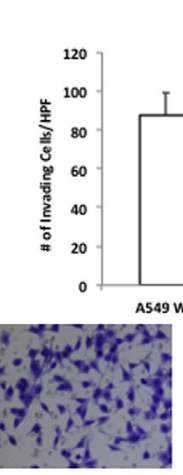

WT
A549

FIGURE 4. shRNA knockdown of Cten expression limits invasion in human lung cancer cells. The effects of reduced Cten expression on lung cancer cells invasion capacity were assessed by transwell invasion assay among untransfected cells, cells transfected with control, nontargeting shRNA, and cells transfected with shRNA targeting Cten expression. Invasion was significantly reduced in Cten knockdown cells relative to controls in both (A) H125 cells $(P=.0036)$ and (B) A549 cells $(P=.006)$. Histograms depict the average number of invading cells per high-power field with associated representative photomicrographs at $20 \times$ magnification below. $\mathrm{n}=3$ for all experiments. $H P F$, High-power field; $K D$, knockdown vector; $N T$, nontargeting control; $W T$, wild type control. 
reduction was significant in both the H125 $(P=.0036)$ and A549 $(P=.006)$ cells (Figure 4$)$.

\section{DISCUSSION}

The results of this study demonstrate for the first time that Cten may play a role in the invasive properties of lung cancer cells in vitro. These data also show that the expression of Cten is upregulated by EGF in lung cancer cells. This upregulation of Cten appears to be at least partly mediated through the STAT3 pathway. This study highlights the potential importance of Cten expression in invasion of non-small cell lung cancer cells.

STAT3 acts as a key transcription factor leading to cancer progression through upregulation of downstream target genes, which have been shown to drive cellular survival, proliferation, and angiogenesis pathways. STAT3 also has been proposed as a mediator of tumor progression in EGFR mutant lung cancer. STAT3 activity and Cten expression both have been independently implicated in cancer cell invasion through their effects on focal adhesion complexes. In a study of glioblastoma cells, Zheng and colleagues ${ }^{16}$ demonstrated that inhibition of the STAT3 pathway significantly reduced cellular motility and invasion. Furthermore, they also demonstrated a decrease in focal adhesion formation. ${ }^{16}$ Likewise, knockdown of Cten in breast cancer cells resulted in a decrease in focal adhesion formation. ${ }^{17}$ The decreased focal adhesion formation that Zheng and colleagues potentially demonstrated with STAT3 inhibition was due to a decrease in Cten expression, but the exact mechanism resulting in fewer focal adhesions remains to be identified. Also, both increased Cten expression and STAT3 activity have been shown to decrease the expression of E-cadherin. ${ }^{10,18}$ Loss of E-cadherin is associated with epithelial to mesenchymal transition and increased migration and invasion of cancer cells.

Although this study shows that STAT3 plays a role in the expression of Cten in lung cancer cells, it is obviously not the only pathway contributing to Cten expression. As demonstrated in the current study, chemical inhibition of STAT3 limited Cten expression in 3 but not all 4 of the cell lines investigated. It may be that other pathways also are involved. Stimulation of cancer cells with EGF activates a host of downstream signaling pathways, one of which is the STAT3 pathway. In colon cancer cells, inhibition of extracellular regulated kinase $1 / 2$ and protein kinase $B$ pathways reduced protein expression of Cten. ${ }^{13}$ It is possible that the A549 cell line does not rely as strongly on the STAT3 pathway for regulating Cten expression, and the extracellular signal-related kinase $1 / 2$ or protein kinase B pathway may play a larger role. Another potential criticism of this study is the use of a single inhibition method to evaluate the role of STAT3 pathway in Cten expression. The highly specific STAT3 inhibitor used in this study,
S3i-201, functions by blocking the binding of STAT3 to its DNA targets, ultimately preventing its transcription factor activity. S3i-201 exerts its inhibitive properties downstream of the phosphorylation of tyrosine and serine residues needed for STAT3 activation. ${ }^{19,20}$ With these data, we demonstrate an activation of the serine residue of STAT3. STAT3 activation requires an initial phosphorylation of tyrosine residues that allows homodimerization of STAT3, which is then able to translocate to the nucleus to function as a transcription factor. The subsequent phosphorylation of a serine residue allows for maximal transcriptional activity of STAT3. ${ }^{12,14}$

In a number of studies evaluating Cten expression in human tumors, increased Cten expression has been found in tumors relative to corresponding normal tissue from melanoma breast, colon, stomach, pancreas, thymus, and lung cancer. Also, increased Cten expression has been shown to positively correlate with tumor staging and negatively correlate with survival in a number of these tissues. ${ }^{3}$ Of note, Cten expression has been shown to be reduced in specimens from prostate and renal cancer relative to the normal tissue counterparts. ${ }^{21-23}$ Although much work has been done establishing a link between Cten expression and tumor progression, the mechanisms by which Cten mediates these changes remain elusive. Liao and colleagues $^{9}$ showed that Cten co-localizes with $\beta$-catenin in the nucleus in colon cancer cells. This nuclear localization of $\beta$-catenin is a key step in the canonical Wnt pathway, which drives transcriptional activity of cellular proliferation and survival pathways. ${ }^{9}$ Albasri and colleagues ${ }^{24}$ demonstrated in colon cancer cells that Cten expression correlated cellular migration through its effects on integrin-linked kinase, a focal adhesion complex signaling protein. Also, Katz and colleagues ${ }^{25}$ demonstrated that upregulation of Cten resulted in a corresponding decrease in tensin 3 and increased cellular migration in breast cells. As previously mentioned, Cten and tensin 3 are both members of the tensin family and bind to integrins as part of focal adhesion complexes. These 2 proteins differ in their ability to bind cytoskeletal actin. Because Cten lacks an actin-binding domain, the resulting lack of interaction between the cytoskeleton and the focal adhesions may allow for these malignant properties. Continued investigation of Cten's role in driving these migratory and invasive changes is merited.

Metastasis and recurrence remain key dilemmas in the treatment of non-small cell lung cancer. Patients who are fortunate enough to be diagnosed early and receive surgical resection of the tumor still face a substantial risk of developing recurrent and metastatic disease. Identification of potential therapeutic targets and development of chemotherapeutics against those targets could improve survival for patients with non-small cell lung cancer. Cten has been shown to play a role in the invasive properties of other solid 
organ tumors, but has not been investigated in non-small cell lung cancer.

\section{CONCLUSIONS}

The results of this present study demonstrate that Cten expression contributes to the invasion of human non-small cell lung cancer cells. Cten expression is at least partly mediated through the STAT3 pathway. Cten should be considered a potential therapeutic target in non-small cell lung cancer.

\section{References}

1. Jemal A, Siegel R, Xu J, Ward E. Cancer Statistics, 2010. CA Cancer J Clin. 2010;60:277-300.

2. Carragher NO, Frame MC. Focal adhesion and actin dynamics: a place where kinases and proteases meet to promote invasion. Trends Cell Biol. 2004;14: 241-9.

3. Lo SH. C-terminal tensin-like (CTEN): a promising biomarker and target for cancer. Int J Biochem Cell Biol. 2014;51C:150-4.

4. Sjoestroem C, Khosravi S, Zhang G, Martinka M, Li G. C-terminal tensin-like protein is a novel prognostic marker for primary melanoma patients. PLoS One. 2013;8:e80492.

5. Albasri A, Aleskandarany M, Benhasouna A, Powe DG, Ellis IO, Ilyas M, et al. CTEN (C-terminal tensin-like), a novel oncogene overexpressed in invasive breast carcinoma of poor prognosis. Breast Cancer Res Treat. 2011;126:47-54.

6. Sasaki H. Cten mRNA expression was correlated with tumor progression in lung cancers. Lung Cancer. 2003;40:151-5.

7. Barbieri I, Pensa S, Pannellini T, Quaglino E, Maritano D, Demaria M, et al. Constitutively active Stat 3 enhances neu-mediated migration and metastasis in mammary tumors via upregulation of Cten. Cancer Res. 2010;70:2558-67.

8. Mouneimne G, Brugge JS. Tensins: a new switch in cell migration. Dev Cell. 2007:13:317-9.

9. Liao Y-C, Chen N-T, Shih Y-P, Dong Y, Lo SH. Up-regulation of C-terminal tensin-like molecule promotes the tumorigenicity of colon cancer through beta-catenin. Cancer Res. 2009;69:4563-6.

10. Albasri A, Seth R, Jackson D, Benhasouna A, Crook S, Nateri S, et al. C-terminal Tensin-like (CTEN) is an oncogene which alters cell motility possibly through repression of E-cadherin in colorectal cancer. J Pathol. 2009;218:57-65.

11. Alvarez JV, Greulich H, Sellers WR, Meyerson M, Frank D. Signal transducer and activator of transcription 3 is required for the oncogenic effects of non-small-cell lung cancer-associated mutations of the epidermal growth factor receptor. Cancer Res. 2006;66:3162-8.

12. Lai SY, Johnson FM. Defining the role of the JAK-STAT pathway in head and neck and thoracic malignancies: implications for future therapeutic approaches. Drug Resist Updat. 2010;13:67-78.

13. Hung S-Y, Shih Y-P, Chen M, Lo SH. Up-regulated Cten by FGF2 contributes to FGF2-mediated cell migration. Mol Carcinog. 2014;53:787-92.

14. Wen Z, Zhong Z, Darnell JE. Maximal activation of transcription by Stat1 and Stat3 requires both tyrosine and serine phosphorylation. Cell. 1995;82:241-50.

15. Gao SP, Mark KG, Leslie K, Pao W, Motoi N, Gerald WL, et al. Mutations in the EGFR kinase domain mediate STAT3 activation via IL-6 production in human lung adenocarcinomas. J Clin Invest. 2007;117:3846-56.

16. Zheng Q, Han L, Dong Y, Tian J, Huang W, Liu Z, et al. JAK2/STAT3 targeted therapy suppresses tumor invasion via disruption of the EGFRvIII/JAK2/STAT3 axis and associated focal adhesion in EGFRvIII-expressing glioblastoma. Neuro Oncol. 2014;0:1-15.

17. Cao X, Voss C, Zhao B, Kaneko T, Li SS. Differential regulation of the activity of deleted in liver cancer 1 (DLC1) by tensins controls cell migration and transformation. Proc Natl Acad Sci U S A. 2012;109:1455-60.

18. Xiong H, Hong J, Du W, Lin Y, Ren L, Wang Y, et al. Roles of STAT3 and ZEB1 proteins in E-cadherin down-regulation and human colorectal cancer epithelialmesenchymal transition. J Biol Chem. 2012;287:5819-32.

19. Sherry M, Reeves A, Wu J, Cochran B. STAT3 is required for proliferation and maintenance of multipotency in glioblastoma stem cells. Stem Cells. 2009;27: 2383-92.

20. Siddiquee K, Zhang S, Guida WC, Blaskovich MA, Greedy B, Lawrence HR, et al. Selective chemical probe inhibitor of Stat3, identified through structurebased virtual screening, induces antitumor activity. Proc Natl Acad Sci U S A 2007; 104:7391-6.

21. Li Y, Mizokami A, Izumi K, Narimoto K, Shima T, Zhang J, et al. CTEN/tensin 4 expression induces sensitivity to paclitaxel in prostate cancer. Prostate. 2010;70: 48-60.

22. Martuszewska D, Ljungberg B, Johansson M, Landberg G, Oslakovic C, Dahlbäck B, et al. Tensin3 is a negative regulator of cell migration and all four Tensin family members are downregulated in human kidney cancer. PLoS One. 2009;4:e4350.

23. Lo SH, Lo TB. Cten, a COOH-terminal tensin-like protein with prostate restricted expression, is down-regulated in prostate Cancer. Cancer Res. 2002; 62:4217-21.

24. Albasri A, Al-Ghamdi S, Fadhil W, Aleskandarany M, Liao Y-C, Jackson D, et al Cten signals through integrin-linked kinase (ILK) and may promote metastasis in colorectal cancer. Oncogene. 2011;30:2997-3002.

25. Katz M, Amit I, Citri A, Shay T, Carvalho S, Lavi S, et al. A reciprocal tensin-3 cten switch mediates EGF-driven mammary cell migration. Nat Cell Biol. 2007; 9:961-9. 